\title{
Applications of a theorem by Ky Fan in the theory of weighted Laplacian graph energy
}

\author{
Reza Sharafdini*, Alireza Ataei, Habibeh Panahbar \\ Department of Mathematics, Faculty of Science, Persian Gulf University, Bushehr 7516913817, Iran
}

Received: 26 April 2016, Accepted: 9 November 2016

Published online: 17 October 2017.

\begin{abstract}
The energy of a graph $G$ is equal to the sum of the absolute values of the eigenvalues of $G$, which in turn is equal to the sum of the singular values of the adjacency matrix of $G$. Let $X, Y$ and $Z$ be matrices, such that $X+Y=Z$. The Ky Fan theorem establishes an inequality between the sum of the singular values of $Z$ and the sum of the sum of the singular values of $X$ and $Y$. This theorem is applied in the theory of graph energy, resulting in several new inequalities.
\end{abstract}

Keywords: Eigenvalue, energy (of graph), singular value (of matrix), Ky Fan theorem, mean deviation, vertex weight.

\section{Introduction}

In this paper, we are concerned with simple graphs. Let $G=(V, E)$ be a simple graph, with nonempty vertex set $V=\left\{v_{1}, \ldots, v_{n}\right\}$ and edge set $E=\left\{e_{1}, \ldots, e_{m}\right\}$. That is to say, $G$ is a simple $(n, m)$-graph. Let $\omega$ be a vertex weight of $G$, i.e., $\omega$ is a function from the set of vertices of $G$ to the set of positive real numbers. $G$ is called $\omega$-regular if for any $u, v \in V(G), \omega(u)=\omega(v)$. Observe that a well-known vertex weight of a graph is the vertex degree weight assigning to each vertex its degree. Let us denote it by $d e g$.

The diagonal matrix of order $n$ whose $(i, i)$-entry is $\omega\left(v_{i}\right)$ is called the diagonal vertex weight matrix of $G$ with respect to $\omega$ and is denoted by $D_{\omega}(G)$, i.e., $D_{\omega}(G)=\operatorname{diag}\left(\omega\left(v_{i}\right), \ldots, \omega\left(v_{n}\right)\right)$. The adjacency matrix $A(G)=\left(a_{i j}\right)$ of $G$ is a $(0,1)$-matrix defined by $a_{i j}=1$ if and only if the vertices $v_{i}$ and $v_{j}$ are adjacent. Then the matrices $L_{d e g}(G)=D_{d e g}(G)-A(G)$ and $L_{d e g}^{\dagger}(G)=A(G)+D_{d e g}(G)$ are called Laplacian and signless Laplacian matrix of $G$, respectively (see [11], [12], [22], [23], [24] and [25]). Let us generalize these matrices for arbitrary vertex weighted graphs. Let $G$ be a simple graph with the vertex weight $\omega$. Then we shall call the matrices $L_{\omega}(G)=D_{\omega}(G)-A(G)$ and $L_{\omega}^{\dagger}(G)=A(G)+D_{\omega}(G)$ the weighted Laplacian and the weighted signless Laplacian matrix of $G$ with respect to the vertex weight $\omega$, respectively. Let $X=\left\{x_{1}, x_{2}, \ldots, x_{n}\right\}$ be a data set of real numbers. The mean absolute deviation (often called the mean deviation) $\mathrm{MD}(X)$ and variance $\operatorname{Var}(X)$ of $X$ is defined as

$$
\operatorname{MD}(X)=\frac{1}{n} \sum_{i=1}^{n}\left|x_{i}-\bar{x}\right|, \quad \operatorname{Var}(X)=\frac{1}{n} \sum_{i=1}^{n}\left(x_{i}-\bar{x}\right)^{2}
$$

where $\bar{x}=\frac{\sum_{i=1}^{n} x_{i}}{n}$ is the arithmetic mean of the distribution. Note that an easy application of the Cauchy-Schwarz inequality gives that the mean deviation is a lower bound on the standard deviation (see [3]).

$$
\operatorname{MD}(X) \leq \sqrt{\operatorname{Var}(X)}
$$


The mean deviation and variance of $G$ with respect to $\omega$, denoted by $\operatorname{MD}_{\omega}(G)$ and $\operatorname{Var}_{\omega}(G)$, respectively, is defined as

$$
\operatorname{MD}_{\omega}(G)=\operatorname{MD}\left(\omega\left(v_{1}\right), \ldots, \omega\left(v_{n}\right)\right), \quad \operatorname{Var}_{\omega}(G)=\operatorname{Var}\left(\omega\left(v_{1}\right), \ldots, \omega\left(v_{n}\right)\right)
$$

It follows from Eq. (1) that $\operatorname{MD}_{\omega}(G) \leq \sqrt{\operatorname{Var}_{\omega}(G)}$. It is worth mentioning that $\operatorname{Var}_{\mathrm{deg}}(G)$ is well-investigated graph invariant (see [2] and [19]). Let $\lambda_{1}, \lambda_{2}, \ldots, \lambda_{n}$ be eigenvalues of the adjacency matrix $A(G)$ of graph $G$. It is known that $\sum_{i=1}^{n} \lambda_{i}=0$. The notion of the energy $\mathscr{E}(G)$ of an $(n, m)$-graph $G$ was introduced by Gutman in connection with the $\pi$-molecular energy (see [13], [14], [16], [17], [21] and [29]). It is defined as

$$
\mathscr{E}(G)=\sum_{i=1}^{n}\left|\lambda_{i}\right|=n \operatorname{MD}\left(\lambda_{1}, \lambda_{2}, \ldots, \lambda_{n}\right)
$$

Let $M \in \mathbb{C}^{n \times n}$ be Hermitian with singular values $s_{i}(M), i=1,2, \ldots, n$. If $\lambda_{i}(M), i=1,2, \ldots, n$ are eigenvalues of $M$, then $s_{i}(M)=\left|\lambda_{i}(M)\right|, i=1,2, \ldots, n$. Getting motivated from this fact, Nikiforov established the concept of matrix energy by analogy with graph energy [26]. Let $M \in \mathbb{C}^{n \times n}$ with singular values $s_{i}(M), i=1,2, \ldots, n$. Then the energy of $M$, denoted by $\mathscr{E}(M)$, is defined as $s_{1}(M)+s_{2}(M)+\ldots+s_{n}(M)$. Consequently, if $M \in \mathbb{C}^{n \times n}$ is Hermitian with eigenvalues $\lambda_{1}(M), \lambda_{2}(M), \ldots, \lambda_{n}(M)$, we have

$$
\mathscr{E}(M)=\sum_{i=1}^{n}\left|\lambda_{i}(M)\right|
$$

Let $n \geq \mu_{1}, \mu_{2}, \ldots, \mu_{n}=0$ be eigenvalues of Laplacian matrix $L(G)$ of an $(n, m)$-graph $G$. It is known that $\sum_{i=1}^{n} \mu_{i}=2 m$. Gutman and Zhou defined the Laplacian energy of an $(n, m)$-graph $G$ for the first time (see [18] ) as

$$
L E(G)=\sum_{i=1}^{n}\left|\mu_{i}-\frac{2 m}{n}\right|=n \mathrm{MD}\left(\mu_{1}, \ldots, \mu_{n}\right)
$$

Numerous results on the Laplacian energy have been reported, see for instance [1], [4], [7], [15], [27], [28] and [34]. Note that in the definition of Laplacian energy $\frac{2 m}{n}$ is the average vertex degree of $G$. This motivates us to extend their definition to the graphs equipped with arbitrary vertex weights. Let $G$ be a graph with the vertex set $V=\left\{v_{1}, \ldots, v_{n}\right\}$ and with an arbitrary vertex weight $\omega$. Let $\mu_{1}, \mu_{2}, \ldots, \mu_{n}$ be eigenvalues of the vertex weighted Laplacian matrix $L_{\omega}(G)$ of graph $G$ with respect to the vertex weight $\omega$. Then we propose the weighted Laplacian energy $L E_{\omega}(G)$ of $G$ with respect to the vertex weight $\omega$ as

$$
L E_{\omega}(G)=\sum_{i=1}^{n}\left|\mu_{i}-\bar{\omega}\right|=n \mathrm{MD}\left(\mu_{1}, \ldots, \mu_{n}\right)
$$

where

$$
\bar{\omega}=\frac{\sum_{i=1}^{n} \omega\left(v_{i}\right)}{n} \quad \text { and } \quad \sum_{i=1}^{n} \mu_{i}=n \bar{\omega}
$$

Note that $L E_{\text {deg }}(G)=L E(G)$.

Let $I_{s}$ be the unit matrix of order $s$. For the considerations that follow it will be necessary to note that instead via Eq. (2), the weighted Laplacian energy can be expressed also as

$$
L E_{\omega}(G)=\mathscr{E}\left(L_{\omega}(G)-\bar{\omega} I_{n}\right)
$$

The following results are already known. The next lemma is known for the vertex degree weight [5]; Its proof for an arbitrary vertex weight is done in a similar way. 
Lemma 1. Let $G$ be a bipartite graphs with $n$ vertices and with a vertex weight $\omega$. Then $L_{\omega}(G)$ and $L_{\omega}^{\dagger}(G)$ are similar.

Lemma 2. [20, Section 7.1, Ex. 2] If $A=\left(a_{i j}\right)_{i, j=1}^{n}$ is a positive semi-definite matrix and $a_{i i}=0$ for some $i$, then $a_{i j}=$ $0=a_{j i}, j=1, \ldots, n$.

Theorem 1, supporting the concept of matrix energy proposed by Nikiforov, was first obtained by Ky Fan [8] using a variational principle. It also appears in Gohberg and Krein [10] and in Horn and Johnson [20]. No equality case is discussed in these references. Thompson [32,33] employs polar decomposition theorem and inequalities due to Fan and Hoffman [9] to obtain its equality case. Day and So [6] gave the details of a proof for the inequality and the case of equality.

Theorem 1.Let $A$ and $B$ be two complex square matrices of size $n\left(A, B \in \mathbb{C}^{n \times n}\right)$ and let $C=A+B$. Then

$$
\mathscr{E}(C) \leq \mathscr{E}(A)+\mathscr{E}(B)
$$

Moreover the equality in Eq. (4) holds if and only if there exists a unitary matrix $P$ such that PA and PB are both positive semi-definite matrices.

Let $A$ be a complex matrix of size $n\left(A \in \mathbb{C}^{n \times n}\right)$. Let us denote the Hermitian adjoint of $A$ by $A^{*}$. Then both $A^{*} A$ and $A A^{*}$ are Hermitian positive semi-definite matrices with the same non-zero eigenvalues. In particular $A^{*} A$ and $A A^{*}$ are diagonalizable with real non-negative eigenvalues. Then by spectral theorem for complex matrices we may define $|A|:=$ $\left(A^{*} A\right)^{1 / 2}$. Here we present the following version of the polar decomposition theorem [20].

Theorem 2. For $A \in \mathbb{C}^{n \times n}$, there exist positive semi-definite matrices $X, Y \in \mathbb{C}^{n \times n}$ and unitary matrices $P, F \in \mathbb{C}^{n \times n}$ such that $A=P X=Y F$. Moreover, the matrices $X, Y$ are unique, $X=|A|, Y=\left|A^{*}\right|$. The matrices $P$ and $F$ are uniquely determined if and only if $A$ is non-singular.

There is a great deal of analogy between the properties of $\mathscr{E}(G)$ and $L E_{\omega}(G)$, but also some significant differences. These similarities and dissimilarities has been investigated [31]. In this paper we apply Theorem 1 in the theory of graph energy, resulting in several new inequalities, as well as new proofs of some earlier known inequalities. It is worth mentioning that the idea of this paper inspired from [27] and [28]; Our proofs are based on those of these references.

\section{Graphs $G$ for which $L E_{\omega}(G)=\mathscr{E}(G)+\mathscr{E}\left(D_{\omega}(G)-\bar{\omega} I_{n}\right)$}

In the case of vertex degree weight, the inequality in the following theorem was proved in [28], whereas the equality in Eq. (5) was investigated in [27]. Based on their proof, we generalize their results for a connected graph with an arbitrary vertex weight.

Theorem 3. Let $G$ be a connected graph with $n$ vertices and with a vertex weight $\omega$. Then

$$
L E_{\omega}(G) \leq n \mathrm{MD}_{\omega}(G)+\mathscr{E}(G)
$$

Moreover the equality in Eq. (5) holds if and if $G$ is $\omega$-regular.

Proof. We Know that

$$
L_{\omega}(G)-\bar{\omega} I_{n}=\left(D_{\omega}(G)-\bar{\omega} I_{n}\right)+(-A(G))
$$

Note that $D_{\omega}(G)-\bar{\omega} I_{n}$ is a diagonal matrix whose eigenvalues are $\omega\left(v_{i}\right)-\bar{\omega}, i=1, \ldots, n$. It follows from Theorem 1 that

$$
\sum_{i=1}^{n} s_{i}\left(L_{\omega}(G)-\bar{\omega} I_{n}\right) \leq \sum_{i=1}^{n} s_{i}\left(D_{\omega}(G)-\bar{\omega} I_{n}\right)+\sum_{i=1}^{n} s_{i}(-A(G))
$$


Therefore

$$
L E_{\omega}(G) \leq \sum_{i=1}^{n}\left|\omega\left(v_{i}\right)-\bar{\omega}\right|+\sum_{i=1}^{n}\left|\lambda_{i}(-A(G))\right| .
$$

Then, due to the similarity between $A(G)$ and $-A(G)$, we have $L E_{\omega}(G) \leq n \mathrm{MD}_{\omega}(G)+\mathscr{E}(G)$.

Let $G$ be a $\omega$-regular graph with eigenvalues $\lambda_{1}, \ldots, \lambda_{n}$. Then $\bar{\omega}=\omega\left(v_{i}\right)$ for each $1 \leq i \leq n$ and $L_{\omega}(G)=\bar{\omega} I_{n}-A(G)$. It follows that $\bar{\omega}-\lambda_{1}, \ldots, \bar{\omega}-\lambda_{n}$ are all the eigenvalues of $L_{\omega}(G)$. Therefore, by Eq. (2) we have $L E_{\omega}(G)=\mathscr{E}(G)$.

Conversely, suppose that the equality in Eq. (5) holds. Without loss of generality, we may assume that $\left.\omega\left(v_{1}\right)=\max \left\{\omega\left(v_{i}\right) \mid 1 \leq i \leq n\right)\right\}$. Suppose on the contrary that $G$ is not $\omega$-regular. Therefore

$$
\omega\left(v_{1}\right)>\bar{\omega}
$$

Let $a_{i}:=\omega\left(v_{i}\right)-\bar{\omega}$ for $i=1, \ldots, n$. We have $a_{1}>0$, via Eq. (7). Due to the equality in Eq. (5), we may apply Theorem 1 to Eq. (6). Therefore, there exists a unitary matrix $P$ such that $X=P\left(D_{\omega}(G)-\bar{\omega} I_{n}\right)$ and $Y=P(-A(G))$ are both positive semi-definite. Hence $P^{*} X$ and $P^{*} Y$ are polar decompositions of the matrices $D_{\omega}(G)-\bar{\omega} I_{n}$ and $-A(G)$, respectively. It follows from Theorem 2 that $X=\left|D_{\omega}(G)-\bar{\omega} I_{n}\right|$ and $Y=|A(G)|$. Therefore $X=\operatorname{diag}\left(\left|a_{1}\right|,\left|a_{2}\right|, \ldots,\left|a_{n}\right|\right)$. Setting

$$
P^{*}=\left(\begin{array}{ccc}
q_{11} & \cdots & q_{1 n} \\
\vdots & \ddots & \vdots \\
q_{n 1} & \cdots & q_{n n}
\end{array}\right), \quad A(G)=\left(\begin{array}{cccc}
0 & a_{12} & \cdots & a_{1 n} \\
a_{12} & 0 & \cdots & a_{2 n} \\
\vdots & \ddots & \ddots & \vdots \\
a_{1 n} & a_{2 n} & \cdots & 0
\end{array}\right)
$$

$P^{*} X=D_{\omega}(G)-\bar{\omega} I_{n}$, implies

$$
\left(\begin{array}{ccc}
q_{11} & \cdots & q_{1 n} \\
\vdots & \ddots & \vdots \\
q_{n 1} & \cdots & q_{n n}
\end{array}\right)\left(\begin{array}{ccc}
\left|a_{1}\right| & & \\
& \ddots & \\
& & \left|a_{n}\right|
\end{array}\right)=\left(\begin{array}{ccc}
a_{1} & & \\
& \ddots & \\
& & a_{n}
\end{array}\right)
$$

Then,

$$
\left(\begin{array}{cccc}
\left|a_{1}\right| q_{11} & \left|a_{2}\right| q_{12} & \cdots & \left|a_{n}\right| q_{1 n} \\
\left|a_{1}\right| q_{21} & \left|a_{2}\right| q_{22} & \cdots & \left|a_{n}\right| q_{2 n} \\
\vdots & \vdots & \ddots & \vdots \\
\left|a_{1}\right| q_{n 1} & \left|a_{2}\right| q_{n 2} & \cdots & \left|a_{1}\right| q_{n n}
\end{array}\right)=\left(\begin{array}{cccc}
a_{1} & & \\
& a_{2} & & \\
& \ddots & \\
& & a_{n}
\end{array}\right) .
$$

Equality at first column imposes $q_{11}=1$ and $q_{i 1}=0, i=2, \ldots, n$. It follows that

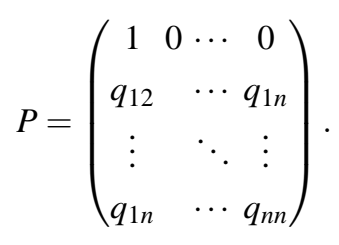


We must then have

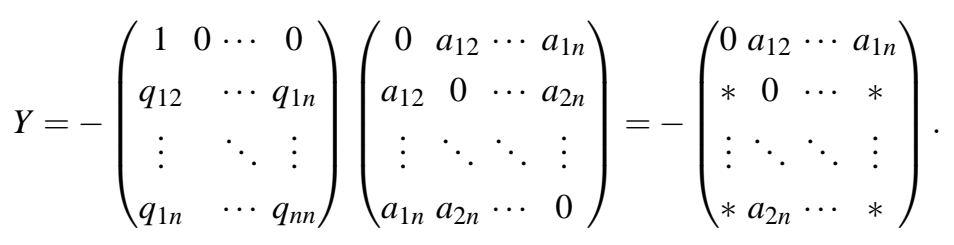

The previous matrix is positive semi-definite and by Lemma 2 , we obtain $a_{1 j}=0, j=2, \ldots, n$. This contradicts our assumption that $G$ is a connected graph and the result follows.

The following is a direct consequence of Theorem 3 .

Corollary 1. Let $G$ be a connected graph with $n$ vertices. Then

$$
\max \left\{L E_{\omega}(G)-n \mathrm{MD}_{\omega}(G) \mid \omega \text { is a vertex weight of } G\right\} \leq \mathscr{E}(G)
$$

3 Graphs $G$ for which $L E_{\omega}(G)=\mathscr{E}(G)$

In Theorem 3 we showed that if $G$ is a $\omega$-regular graph, then $L E_{\omega}(G)=\mathscr{E}(G)$. In what follows we consider the converse argument.

In the case of vertex degree weight, the first part of the following theorem was proved in [28], whereas the second part was proved in [27]. Based on their proof, we generalize their results for a connected graph with an arbitrary vertex weight.

Theorem 4. Let $G$ be a bipartite graph with a vertex weight $\omega$. Then

$$
L E_{\omega}(G) \geq \mathscr{E}(G)
$$

Moreover, the equality in Eq. (8) holds if and only if $G$ is a $\omega$-regular graph.

Proof. From the definition of weighted Laplacian matrix and weighted signless Laplacian matrix, it is clear that

$$
\left(L_{\omega}^{\dagger}(G)-\bar{\omega} I_{n}\right)-\left(L_{\omega}(G)-\bar{\omega} I_{n}\right)=2 A(G)
$$

If $G$ is bipartite, then it follows from Lemma 1 that $L_{\omega}(G)$ and $L_{\omega}^{\dagger}(G)$ have the same spectra and therefore

$$
\sum_{i=1}^{n} s_{i}\left(L_{\omega}^{\dagger}(G)-\bar{\omega} I_{n}\right)=\sum_{i=1}^{n} s_{i}\left(L_{\omega}-\bar{\omega} I_{n}\right)=\sum_{i=1}^{n} s_{i}\left(-\left[L_{\omega}(G)-\bar{\omega} I_{n}\right]\right)=L E_{\omega}(G)
$$

So by Theorem $1, L E_{\omega}(G) \geq \mathscr{E}(G)$.

Let $G$ be a $\omega$-regular graph. Then by Theorem 3, the equality in Eq. (8) holds. Conversely, suppose that the equality in Eq. (8) holds. Therefore,

$$
\mathscr{E}\left(\left(L_{\omega}^{\dagger}(G)-\bar{\omega} I_{n}\right)-\left(L_{\omega}(G)-\bar{\omega} I_{n}\right)\right)=2 \mathscr{E}(G)=\mathscr{E}(G)+\mathscr{E}(G)=L E_{\omega}(G)+L E_{\omega}(G)
$$

Since $G$ is bipartite it follows from Lemma 1 that

$$
\mathscr{E}\left(\left(L_{\omega}^{\dagger}(G)-\bar{\omega} I_{n}\right)-\left(L_{\omega}(G)-\bar{\omega} I_{n}\right)\right)=\mathscr{E}\left(L_{\omega}^{\dagger}(G)-\bar{\omega} I_{n}\right)+\mathscr{E}\left(-\left(L_{\omega}(G)-\bar{\omega} I_{n}\right)\right)
$$


Therefore, Theorem 1 asserts that there exists a unitary matrix $P$, such that

$$
X=P\left(L_{\omega}^{\dagger}(G)-\bar{\omega} I_{n}\right) \text { and } Y=P\left(-\left(L_{\omega}(G)-\bar{\omega} I_{n}\right)\right)
$$

are both positive semi-definite matrices. Hence $P^{*} X$ and $P^{*} Y$ are polar decompositions of $L_{\omega}^{\dagger}(G)-\bar{\omega} I_{n}$ and $-\left(L_{\omega}(G)-\bar{\omega} I_{n}\right)$, respectively. By Theorem 1 we obtain $X=\left|L_{\omega}^{\dagger}(G)-\bar{\omega} I_{n}\right|$ and $Y=\left|-\left(L_{\omega}(G)-\bar{\omega} I_{n}\right)\right|$.

In view of the fact that $G$ is bipartite, we conclude that $X=Y$. Therefore, it follows from Eq. (11) that

$$
L_{\omega}^{\dagger}(G)+L_{\omega}(G)=2 \bar{\omega} I_{n}
$$

implying the result.

The following is a direct consequence of Theorem 4.

Corollary 2. For a bipartite graph $G$ we have

$$
\min \left\{L E_{\omega}(G) \mid \omega \text { is a vertex weight of } G\right\} \geq \mathscr{E}(G)
$$

In the case of vertex degree weight, the next theorem was proved in [28] and based on their proof, we get also the following theorem.

Theorem 5. Let $G$ be a bipartite graph with $n$ vertices and with a vertex weight $\omega$. Then

$$
\max \left\{n \mathrm{MD}_{\omega}(G), \mathscr{E}(G)\right\} \leq L E_{\omega}(G) \leq n \mathrm{MD}_{\omega}(G)+\mathscr{E}(G)
$$

Proof. The right side inequality is a direct consequent of Theorem 3. Let us prove the left one. It is easy to see that

$$
L_{\omega}^{\dagger}(G)+L_{\omega}(G)=2 D_{\omega}(G)
$$

from which $\left(L_{\omega}^{\dagger}(G)-\bar{\omega} I_{n}\right)+\left(L_{\omega}(G)-\bar{\omega} I_{n}\right)=2\left(D_{\omega}(G)-\bar{\omega} I_{n}\right)$. It follows from Theorem 1 that

$$
\mathscr{E}\left(L_{\omega}^{\dagger}(G)-\bar{\omega} I_{n}\right)+\mathscr{E}\left(L_{\omega}(G)-\bar{\omega} I_{n}\right) \geq 2 \mathscr{E}\left(D_{\omega}(G)-\bar{\omega} I_{n}\right)=2 n \mathrm{MD}_{\omega}(G)
$$

In the other hand, since $G$ is bipartite, it follows from Lemma 1 that $L E_{\omega}(G)=\mathscr{E}\left(L_{\omega}^{\dagger}(G)-\bar{\omega} I_{n}\right)=\mathscr{E}\left(L_{\omega}(G)-\bar{\omega} I_{n}\right)$. Therefore

$$
L E_{\omega}(G) \geq n \mathrm{MD}_{\omega}(G)
$$

Hence, the result follow from Eq. (13) and Theorem 4.

\section{An upper bound on the Laplacian matrix energy for the disjoint union of graphs}

Here and throughout this section, $\bigoplus$ denotes the block matrix direct sum [20]. Let $k \in \mathbb{N}$. Suppose that for each $1 \leq i \leq k, G_{i}=\left(V_{i}, E_{i}\right)$ is an $\left(n_{i}, m_{i}\right)$-graph with the vertex set $V_{i}$ and the edge set $E_{i}$. Let $V_{i}$ 's are mutually disjoint. In this case the disjoint union of $G_{i}$ 's, denoted by $\bigcup_{i=1}^{k} G_{i}$, is a non-connected graph with the vertex set $\bigcup_{i=1}^{k} V_{i}$ and the edge set $\bigcup_{i=1}^{k} E_{i}$. It is easy to see that $A\left(\bigcup_{i=1}^{k} G_{i}\right)=\bigoplus_{i=1}^{k} A\left(G_{i}\right)$. 
Moreover, if $\omega_{i}$ is a vertex weight, assigned to $G_{i}$, then $\bigcup_{i=1}^{k} G_{i}$ inherits naturally a vertex weight from its components. This weight is nothing but $\omega:=\bigcup_{i=1}^{k} \omega_{i}$, i.e., For each $v \in \bigcup_{i=1}^{k} V_{i}, \omega(v)=\omega_{i}(v)$ if and only if $v \in V_{i}$. Note that $\bar{\omega}$ is a convex combination of $\bar{\omega}_{i}, i=1, \ldots, k$, since

$$
\bar{\omega}=\left(\frac{1}{\sum_{j=1}^{k} n_{j}}\right)\left(\sum_{i=1}^{k} \sum_{v \in V_{i}} \omega_{i}(v)\right)=\sum_{i=1}^{k}\left(\frac{n_{i}}{\sum_{j=1}^{k} n_{j}}\right) \bar{\omega}_{i}
$$

Moreover $\bar{\omega} \geq \overline{\omega_{i}}, \quad i=1, \ldots, k$

In the case of vertex degree weight, the next theorem was proved in [27] and based on their proof, we get also the following result.

Theorem 6. Let $k \in \mathbb{N}$. Suppose that for each $1 \leq i \leq k, G_{i}$ is a graph with $n_{i}$ vertices and with a vertex weight $\omega_{i}$. Then

$$
L E_{\omega}\left(\bigcup_{i=1}^{k} G_{i}\right) \leq \sum_{i=1}^{k} L E_{\omega_{i}}\left(G_{i}\right)+\sum_{i=1}^{k}\left|\bar{\omega}_{i}-\bar{\omega}\right| n_{i}
$$

Equality holds if and only if $\bar{\omega}_{i}=\bar{\omega}$ for all $i=1, \ldots, k$.

Proof. In order to simplify the writing and omit some subscripts, for each $1 \leq i \leq k$, we denote $I_{n_{i}}$ and $\bar{\omega}_{i}-\bar{\omega}$ by $I_{i}$ and $b_{i}$, respectively. It is clear that

$$
L_{\omega}(G)-\bar{\omega} I_{n}=\bigoplus_{i=1}^{k}\left(L_{\omega_{i}}\left(G_{i}\right)-\bar{\omega} I_{i}\right)=\bigoplus_{i=1}^{k}\left(L_{\omega_{i}}\left(G_{i}\right)-\bar{\omega}_{i} I_{i}\right)+\bigoplus_{i=1}^{k} b_{i} I_{i}
$$

Therefore, as a consequence of Eq. (3) and Theorem 1, the inequality in Eq. (15) follows.

Now let us consider the the equality case in Eq. (15). Let $\bar{\omega}_{i}=\bar{\omega}$ for all $i=1, \ldots, k$. Therefore the matrix $\bigoplus_{i=1}^{k} b_{i} I_{i}$ is zero and consequently it follows from Eq. (16) that the equality in Eq. (15) holds.

Conversely suppose on the contrary that there exists $1 \leq l \leq k$ such that $\bar{\omega}_{l}>\bar{\omega}$. We may assume that $l=1$. As a consequence of Theorem 1, Eq. (16) and the equality in Eq. (15), there exists a unitary matrix $P$ such that

$$
X=P \bigoplus_{i=1}^{k}\left(L_{\omega_{i}}\left(G_{i}\right)-\bar{\omega}_{i} I_{i}\right) \text { and } Y=P \bigoplus_{i=1}^{k} b_{i} I_{i}
$$

are both positive semi-definite. Hence $P^{*} X$ and $P^{*} Y$ are polar decompositions of the matrices

$$
\bigoplus_{i=1}^{k}\left(L_{\omega_{i}}\left(G_{i}\right)-\bar{\omega}_{i} I_{i}\right) \text { and } \bigoplus_{i=1}^{k} b_{i} I_{i}
$$

respectively. By Theorem 2, we arrive at

$$
Y=\bigoplus_{i=1}^{k}\left|b_{i}\right| I_{i}=P \bigoplus_{i=1}^{k} b_{i} I_{i}
$$


We can write the unitary matrix $P$ as

$$
P=\left(\begin{array}{cccc}
P_{11} & P_{12} & \cdots & P_{1 k} \\
P_{21} & P_{22} & \cdots & P_{2 k} \\
\vdots & & \ddots & \vdots \\
P_{k 1} & \cdots & & P_{k k}
\end{array}\right)
$$

with the diagonal matrices $P_{j j}, j=1, \ldots, k$ of order $n_{j}$, respectively. From Eq. (17) we have

$$
\left(\begin{array}{cccc}
\left|b_{1}\right| I_{1} & 0 & \cdots & 0 \\
0 & \left|b_{2}\right| I_{2} & \cdots & 0 \\
\vdots & & \ddots & \vdots \\
0 & \cdots & 0 & \left|b_{k}\right| I_{k}
\end{array}\right)=\left(\begin{array}{cccc}
P_{11} & P_{12} & \cdots & P_{1 k} \\
P_{21} & P_{22} & \cdots & P_{2 k} \\
\vdots & & \ddots & \vdots \\
P_{k 1} & \cdots & & P_{k k}
\end{array}\right)\left(\begin{array}{cccc}
b_{1} I_{1} & 0 & \cdots & 0 \\
0 & b_{2} I_{2} & \cdots & 0 \\
\vdots & & \ddots & \vdots \\
0 & \cdots & 0 & b_{k} I_{k}
\end{array}\right)
$$

and then

$$
\left(\begin{array}{cccc}
\left|b_{1}\right| I_{1} & 0 & \cdots & 0 \\
0 & \left|b_{2}\right| I_{2} & \cdots & 0 \\
\vdots & & \ddots & \vdots \\
0 & \cdots & 0 & \left|b_{k}\right| I_{k}
\end{array}\right)=\left(\begin{array}{cccc}
b_{1} P_{11} & P_{12} & \cdots & P_{1 k} \\
b_{1} P_{21} & P_{22} & \cdots & P_{2 k} \\
\vdots & & \ddots & \vdots \\
b_{1} P_{k 1} & \cdots & & P_{k k}
\end{array}\right)
$$

As $b_{1}=\bar{\omega}_{1}-\bar{\omega}>0$, via Eq. (19) we obtain $P_{11}=I_{1}$ and $P_{j 1}=0, j=2, \ldots, k$. Now it follows from $X=P \bigoplus_{i=1}^{k}\left(L_{\omega_{i}}\left(G_{i}\right)-\bar{\omega}_{i} I_{i}\right)$ that $L_{\omega_{1}}\left(G_{1}\right)-\bar{\omega}_{1} I_{1}$ is positive semi-definite. Now we have the required contradiction, since by the Rayleigh principle we find that $L_{\omega_{1}}\left(G_{1}\right)-\bar{\omega}_{1} I_{1}$ has a negative eigenvalue. Hence the assertion follows.

\section{Conclusion}

The vertex degree may be considered as a vertex weight of a graph. Inspired from this we just replaced the vertex degree by an arbitrary vertex weight in the definition of Laplacian matrix and investigated the differences and similarities with the results in [27] where the vertex degree weight was considered. What we obtained shows that our results generalize most of those in [27]. To support our theory we refer to [30] where several natural vertex weights were considered.

\section{Acknowledgements}

The authors are grateful to anonymous referees for their careful reading the manuscript and their valuable comments. One of the referees suggested to include Corollary 1 and Corollary 2. We acknowledge his/her contribution which improved the presentation of the manuscript.

\section{Competing interests}

The authors declare that they have no competing interests.

\section{Authors' contributions}

All authors have contributed to all parts of the article. All authors read and approved the final manuscript. 


\section{References}

[1] Aleksic, T. Upper bounds for Laplacian energy of graphs. MATCH Commun. Math. Comput. Chem. 2008, 60, 435-439.

[2] Bell, F.K. A Note on the Irregularity of Graphs. Linear Algebra Appl. 1992, 161,45-54.

[3] Cavers, M.S. The normalized laplacian matrix and general randic index of graphs. Ph.D. Thesis, University of Regina, Regina, Saskatchewan, 2010.

[4] Das, C.K.; Mojallala, S.A.; Gutman, I. On energy and Laplacian energy of bipartite graphs. Appl. Math. Comput. 2016, 273, 759-766.

[5] Cvetkovic; Doob, M.; Sachs, H. Spectra of Graphs-Theory and Application. third ed.; Johann Ambrosius Barth Verlag, Heidelberg: Leipzig, 1995.

[6] Day, J.; So, W. Singular value inequality and graph energy change. El. J. Linear Algebra 2007, 16, 291-297.

[7] Abreu, N.N.M. de ; Vinagre, C.M.; Bonifacio, A.S.; Gutman, I. The Laplacian energy of some Laplacian integral graphs. MATCH Commun. Math. Comput. Chem. 2008, 60, 447-460.

[8] Fan, K. Maximum properties and inequalities for the eigenvalues of completely continuous operators. Proc. Nat. Acad. Sci.U.S.A. 1951, (37), 760-766.

[9] Fan, K.; Hoffman, A.J. Some metric inequalities in the space of matrices. Proc. Amer. Math. Soc. 1955 6, 111-116.

[10] Gohber, I.; Krein, M. Introduction to the Theory of Linear Nonselfadjoint Operators. Amer. Math. Soc. Providence. 1969.

[11] Grone, R.; Merris, R. The Laplacian spectrum of a graph II. SIAM J. Discrete Math. 1994, 7, 221-229.

[12] Grone, R.; Merris, R.; Sunder, V.S. The Laplacian spectrum of a graph. SIAM J. Matrix Anal. Appl. 1990, 11, $218-238$.

[13] Gutman, I. The energy of a Graph, Old and New Results. In Algebraic Combinatorics and Applications; A., Betten.; A., Kohnert.; R., Laue.; A., Wassermann.;Eds.; Springer-Verlag: Berlin, 2001; pp. 196-211.

[14] Gutman, I. The energy of a graph. Ber. Math.-Statist. Sekt. Forschungsz. Graz. 1978, 103, 1-22.

[15] Gutman, I.; Abreu, N.M.M. de; Vinagre, C.T.M.; Bonifacio, A.S.; Radenkovic, S. Relation between energy and Laplacian energy. MATCH Commun. Math. Comput. Chem. 2008, 59, 343-354.

[16] Gutman, I.; Polansky, O.E. Mathematical Concepts in Organic Chemistry. Springer-Verlag: Berlin, 1986; Chapter 8.

[17] Gutman, I.; Zare Firoozabadi, S.; de la Pena, J.A.; Rada, J. On the energy of regular graphs. MATCH Commun. Math. Comput. Chem. 2007, 57, 435-442.

[18] Gutman, I.; Zhou, B. Laplacian energy of a graph. Linear Algebra Appl. 2006, 414, 29-37.

[19] Gutman, I.; Paule, P. The variance of the vertex degrees of randomly generated graphs. Univ. Beograd. Publ. Elektrotehn. Fak. Ser. Mat. 2002, 13, 30-35.

[20] Horn, R.; Johnson, C. Matrix Analysis. Cambridge Univ. Press: Cambridge, 1989.

[21] Indulal, G.; Vijayakumar, A. A note on energy of some graphs. MATCH Commun. Math. Comput. Chem. 2008, 59, $269-274$.

[22] Merris, R. A survey of graph Laplacians. Linear Multilinear Algebra 1995, 39, 19-31.

[23] Merris, R. Laplacian matrices of graphs: a survey. Linear Algebra Appl. 1994, 197-198, 143-176.

[24] Mohar, B., The Laplacian spectrum of graphs. In Graph Theory, Combinatorics, and Applications; Alavi, Y.; Chartrand, G.; Oellermann, O.R.; Schwenk, A.J., Eds.; Wiley: New York, 1991; pp. 871-898.

[25] Mohar, B., Graph Laplacians. In Topics in Algebraic Graph Theory; Brualdi, L.W.; Wilson, R.J., Eds.; Cambridge Univ. Press: Cambridge, 2004; pp. 113-136.

[26] Nikiforov, V. The energy of graphs and matrices. J. Math. Anal. Appl. 2007, 326, 1472-1475.

[27] Robbiano, M.; Jiménez, R. Applications of a theorem by Ky Fan in the theory of Laplacian energy of graphs. MATCH Commun. Math. Comput. Chem. 2009, 62, 537-552.

[28] So, W.; Robbiano, M.; Abreu, N.M.M. de; Gutman, I. Applications of the Ky Fan theorem in the theory of graph energy. Linear Algebra Appl. 2010, 432, 2163-2169.

[29] Li, X.; Shi, Y.; Gutman I. Graph Energy, Springer, New York, 2012.

[30] Sharafdini, R.; Panahbar, H. Vertex weighted Laplacian graph energy and other topological indices. J. Math. Nanosci. 2016, 6, 49-57.

[31] Sharafdini, R.; Panahbar, H. On the weighted version of Laplacian energy of graphs. manuscript. 
331 BSM R. Sharafdini, A.R. Ataei, H. Panahbar: Applications of a theorem by ky fan in the theory of weighted...

[32] Thompson, R.C. Convex and concave functions of singular values of matrix sums. Pacific J. Math. 1976, 66, $285-290$.

[33] Thompson, R.C. The case of equality in the matrix-valued triangle inequalitity. Pacific J. Math. 1979, 82, $279-280$.

[34] Zhou, B.; Gutman, I.; Aleksic, T. A note on Laplacian energy of graphs. MATCH Commun. Math. Comput. Chem. 2008, 60, 441-446. 